\title{
The dust environment of comet 67P/Churyumov-Gerasimenko*
}

\author{
M. Fulle ${ }^{1}$, C. Barbieri ${ }^{2}$, G. Cremonese ${ }^{3}$, H. Rauer ${ }^{4}$, M. Weiler ${ }^{4}$, G. Milani ${ }^{5}$, and R. Ligustri ${ }^{6}$ \\ 1 INAF - Osservatorio Astronomico di Trieste, Via Tiepolo 11, 34131 Trieste, Italy \\ 2 Dipartimento di Astronomia, Università di Padova, Vicolo dell'Osservatorio 2, 35122 Padova, Italy \\ 3 INAF - Osservatorio Astronomico di Padova, Vicolo dell'Osservatorio 5, 35122 Padova, Italy \\ ${ }^{4}$ Institute of Planetary Research, DLR Rutherfordstrasse 2, 12849 Berlin, Germany \\ 5 Unione Astrofili Italiani, UAI \\ ${ }^{6}$ Osservatorio di Talmassons, CAST, Talmassons (Ud), Italy
}

Received 4 December 2003 / Accepted 6 April 2004

\begin{abstract}
The new target of the ESA Rosetta Mission is comet 67P/Churyumov-Gerasimenko, which passed its last perihelion on 18 August 2002 and was well observed from fall 2002 to spring 2003. Its most prominent feature was a thin dust tail, which is best fitted by the Neck-Line model. Fits of the whole tail provide the dust environment of 67P during a year around perihelion; it shows a strong asymmetry between pre and post perihelion times. The dust mass loss rate appears constant since $2 \mathrm{AU}$ before perihelion at about $200 \mathrm{~kg} \mathrm{~s}^{-1}$, a factor 100 higher than 46P/Wirtanen, the previous Rosetta target. Neck-Line photometry during 2002 and 2003 suggests that such a dust environment has remained similar since 3.6 AU before perihelion, i.e. the distance at which Rosetta science operations will start and the lander will be delivered to the surface.
\end{abstract}

Key words. space vehicles - comets: general - comets: individual: 67P/Churyumov-Gerasimenko

\section{Introduction}

Due to a launch delay, in February 2003 ESA changed the target of the Rosetta Mission (ESA 2003): from 46P/Wirtanen to $67 \mathrm{P} /$ Churyumov-Gerasimenko (67P hereafter for brevity). A strong effort was required to obtain information on the new target in order to change the orbiting strategies to adapt the already built spacecraft and instruments to an environment possibly different from the planned one: this regards not only the shape and size of the nucleus, but also the coma environment, which is related to shape and spin state of the nucleus. Here we focus on the dust environment of 67P: a comparison with the environment of the previous target $46 \mathrm{P}$ will allow us to outline the differences between two well studied short-period comets, in order to understand if $67 \mathrm{P}$ is representative of the family of short-period comets. Moreover, a model of the dust environment is necessary to plan the operations of the many instruments studying the dust ejected from the comet, to determine the orbiting strategies, the software robustness of the navigation cameras against false nucleus detection, and the lifetime of the landing probe against dust pollution.

In this paper we consider IRAS observations performed in 1983 (Sykes \& Walker 1992) and ground-based data collected on the dust ejected from 67P during the nine months of

Send offprint requests to: M. Fulle, e-mail: fulle@ts . astro.it

* Based also on observations collected at the National Galileo Telescope and the $2 \mathrm{~m}$ telescope of the Thüringer Landessternwarte Tautenburg. observability after the perihelion passage of August 18, 2002: before August 2002 and after spring 2003, the comet was too close to the Sun to be observable. We will analyse in another paper the data collected before the 1996 perihelion passage. During 2002 and 2003, 67P showed a 10 arcmin long and thin tail (Fig. 1), bright enough to be monitored by CCD cameras of amateur astronomers. This is unusual for short-period comets, which rarely show bright tails. We will show in this paper that, certainly during all spring 2003, and probably also during fall 2002 , this tail was a dust tail. If ground-based observations only are available, models of dust tails provide the most complete information on a comet dust environment (Fulle 1999). Coma photometry, quantified by the quantity Af $\rho$ (A'Hearn et al. 1984), will then be used to constrain the outputs of dust tail models. These suggest that the dust environment of 67P may be different from that of typical short-period comets, in which such a long lasting dust tail was never observed. Therefore, a deep analysis of the dust environment of 67P is even more important in the perspective of the Rosetta Mission.

Let us recall that one of the difficulties in interpreting dust images is that the dust ejections occurring days before, months before or years before the time of observation form patterns which may have a similar appearance. Therefore, extreme care must be paid to obtain a correct identification. If an observed pattern is improperly identified, the derived time (and in general the rate) of dust production will be severely in error. In the case of 67P, the identification of the thin tail in terms of a trail (Sykes \& Walker 1992) or in terms of a Neck-Line 
Table 1. The observed Position Angle $\mathrm{PA}_{\mathrm{T}}$ of the Tail is compared with the antisolar direction (Position Angle PA $\mathrm{R}_{\mathrm{RV}}$ of the prolonged radius vector) and the expected position of the dust trail (Position Angle $\mathrm{PA}_{\mathrm{CO}}$ of the comet orbit projected on the sky). The observed $\mathrm{PA}_{\mathrm{T}}$ values are best fit by the computed $\mathrm{PA}_{\mathrm{NL}}$ of the Neck-Line ejected at Sun-Comet distances $r(\theta-\pi)$ and at the $t(\theta-\pi)-T$ times (days with respect to perihelion $T$ ), where $\theta$ is the comet true anomaly at observation. Observers: DT, Diego Tirelli; RL, Rolando Ligustri (Talmassons Observatory); HM, Hermann Mikuz (Crni Vrh Observatory); TNG, National Galileo Telescope; TLS, Tautenburg Schmidt Telescope at Thüringer LandesSternwarte.

\begin{tabular}{|c|c|c|c|c|c|c|c|c|c|c|c|}
\hline Time UT & $\begin{array}{c}t(\theta)-T \\
{[\text { days] }}\end{array}$ & $\begin{array}{c}r(\theta) \\
{[\mathrm{AU}]}\end{array}$ & $\begin{array}{c}\theta \\
{\left[{ }^{\circ}\right]}\end{array}$ & $\begin{array}{c}t(\theta-\pi)-T \\
\quad[\text { days }]\end{array}$ & $\begin{array}{c}r(\theta-\pi) \\
{[\mathrm{AU}]}\end{array}$ & $\begin{array}{c}\mathrm{PA}_{\mathrm{NL}} \\
{\left[^{\circ}\right]}\end{array}$ & $\begin{array}{c}\mathrm{PA}_{\mathrm{CO}} \\
{\left[^{\circ}\right]}\end{array}$ & $\begin{array}{c}\mathrm{PA}_{\mathrm{RV}} \\
{\left[^{\circ}\right]}\end{array}$ & $\begin{array}{c}\mathrm{PA}_{\mathrm{T}} \\
\left.{ }^{\circ}\right]\end{array}$ & Band & Observer \\
\hline 14.094 Aug. 2002 & -4.219 & 1.29 & -3.6 & - & - & - & 263.7 & 272.4 & $270 \pm 3$ & $R$ & RL \\
\hline 8.107 Sep. 2002 & 21.794 & 1.32 & 18.4 & -810 & 5.28 & 275.4 & 274.6 & 283.3 & $280 \pm 3$ & $V$ & DT \\
\hline 19.134 Oct. 2002 & 62.821 & 1.49 & 48.4 & -378 & 3.62 & 289.8 & 288.6 & 293.5 & $290 \pm 3$ & V & DT \\
\hline 8.059 Nov. 2002 & 82.746 & 1.61 & 60.7 & -285 & 3.05 & 294.2 & 293.9 & 295.0 & $294 \pm 3$ & $R$ & RL \\
\hline 11.154 Nov. 2002 & 85.841 & 1.63 & 62.4 & -275 & 2.99 & 294.7 & 294.6 & 295.1 & $296 \pm 3$ & V & DT \\
\hline 4.175 Jan. 2003 & 140.862 & 2.03 & 86.3 & -164.2 & 2.20 & 299.3 & 303.9 & 291.0 & $296 \pm 3$ & $V$ & DT \\
\hline 11.188 Jan. 2003 & 147.875 & 2.08 & 88.7 & -156.4 & 2.14 & 299.3 & 304.3 & 289.6 & $297 \pm 3$ & $R$ & RL \\
\hline 12.197 Jan. 2003 & 148.884 & 2.09 & 89.0 & -155.3 & 2.13 & 299.3 & 304.4 & 289.4 & $295 \pm 3$ & $R$ & RL \\
\hline 2.003 Feb. 2003 & 167.690 & 2.23 & 94.8 & -138.0 & 2.00 & 298.6 & 303.9 & 282.7 & $297 \pm 3$ & $R$ & RL \\
\hline 22.935 Mar. 2003 & 216.622 & 2.58 & 107.0 & -107.7 & 1.78 & 294.6 & 297.6 & 138.7 & $296 \pm 2$ & $R$ & HM \\
\hline 27.000 Mar. 2003 & 220.687 & 2.61 & 107.8 & -105.8 & 1.77 & 294.4 & 297.1 & 133.2 & $294.5 \pm 0.5$ & $R$ & TNG \\
\hline 27.875 Mar. 2003 & 221.562 & 2.62 & 108.0 & -105.4 & 1.76 & 294.3 & 297.0 & 132.2 & $294.0 \pm 0.5$ & $R$ & TLS \\
\hline 28.028 Mar. 2003 & 221.715 & 2.62 & 108.1 & -105.3 & 1.76 & 294.3 & 297.0 & 132.0 & $294.0 \pm 0.5$ & $R$ & TLS \\
\hline 28.896 Mar. 2003 & 222.583 & 2.63 & 108.2 & -104.9 & 1.76 & 294.3 & 296.9 & 131.1 & $294.0 \pm 0.5$ & $R$ & TLS \\
\hline 6.922 Apr. 2003 & 230.609 & 2.69 & 110.1 & -101.0 & 1.73 & 294.0 & 296.2 & 124.5 & $294 \pm 2$ & $R$ & $\mathrm{HM}$ \\
\hline
\end{tabular}

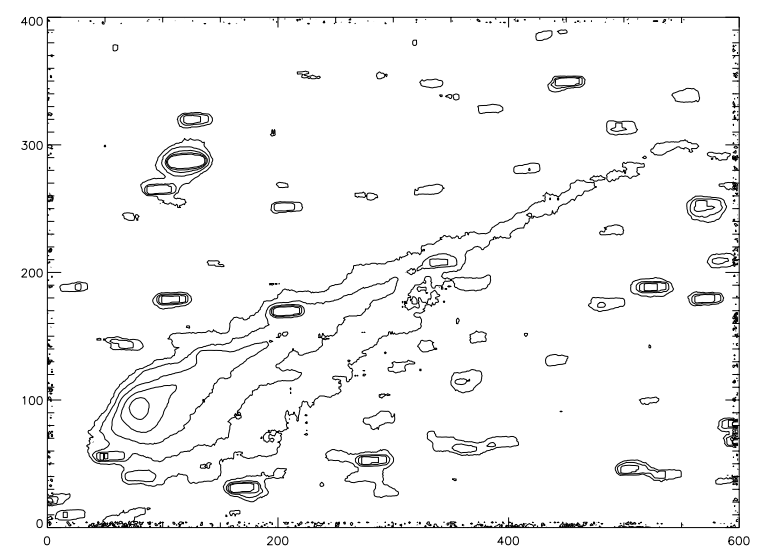

Fig. 1. Image of the thin tail of 67P taken on 27.875 March 2003 UT at the $2 \mathrm{~m}$ Tautenburg Schmidt Telescope (see Table 1 for details of the observations). $67 \mathrm{P}$ showed a similar tail during nine months after 2002 perihelion. The axis units are pixels, with scale 1.23 arcsec pixel $^{-1}$ (the scale reported in Weiler et al. (2004a) is wrong). The image width is $9 \times 10^{5} \mathrm{~km}$ projected at the Earth-comet distance. North is up. The antisolar and tail Position Angles are $132^{\circ}$ and $294^{\circ}$, respectively, both rotating anticlockwise from North $\left(\mathrm{PA}_{\mathrm{RV}}\right.$ and $\mathrm{PA}_{\mathrm{T}}$ in Table 1). A factor two intensity step was chosen between isophotes.

(Kimura \& Liu 1977) might shift the same dust production from 1.3 AU up to 3.6 AU pre-perihelion: the difference is of extreme significance for the Rosetta Mission. We will show that the thin tail analysed here cannot be identified with the IRAS trail, which is orders of magnitude fainter and does not fit the tail position on the sky. We will compare the estimates of the dust loss rates provided by models of dust tails and IRAS trails.

\section{67P Tail: Evolution over nine months}

Databases of the Comet Section of the Italian Amateur Astronomer Union (UAI) contain many CCD images following the evolution of the thin tail during the whole 2002/03 observability window (UAI 2003). These images are filtered using broadband filters only, like Cousins R, and are here complemented by four images of much higher quality obtained at the National Galileo Telescope and the Tautenburg Schmidt Telescope (Weiler et al. 2004a, Fig. 1). The log of all the observations analysed in this paper is reported in Table 1 . Here we report also the correspondence among UT times, the comet true anomaly $\theta$ (i.e. the angle between the Sun-Comet and the Sun-perihelion vectors), the observation time related to perihelion $t(\theta)-T$, and the Sun-Comet distance $r(\theta)$ : in the following sections and figures, Table 1 can be used to convert one of these quantities to the others.

The 67P tail is so thin that it can be described as a linear spike leaving the coma with a sky-projected Position Angle $\mathrm{PA}_{\mathrm{T}}$. This can be compared to the Position Angle $\mathrm{PA}_{\mathrm{RV}}$ of the prolonged radius vector (i.e. the antisolar direction projected on the sky) and to the Position Angle PA $\mathrm{CO}_{\mathrm{CO}}$ of the comet orbit projected on the sky. If the thin tail is a trail, we must have $\mathrm{PA}_{\mathrm{T}}=\mathrm{PA}_{\mathrm{CO}}$. The data in Table 1 allow us to exclude that the thin tail is the IRAS trail. If the tail is an ion tail, $\mathrm{PA}_{\mathrm{T}}$ must be always close to $\mathrm{PA}_{\mathrm{RV}}$. More precisely, the $67 \mathrm{P}$ orbital velocity is less than $35 \mathrm{~km} \mathrm{~s}^{-1}$; the solar wind radial velocity is greater than $400 \mathrm{~km} \mathrm{~s}^{-1}$, while its tangential component is less than $50 \mathrm{~km} \mathrm{~s}^{-1}$ (Brandt et al. 1972); it follows that $\left|\mathrm{PA}_{\mathrm{T}}-\mathrm{PA}_{\mathrm{RV}}\right|<10^{\circ}$. The data in Table 1 show that the tail could have been an ion tail during 2002, but this was impossible during 2003. If $\mathrm{PA}_{\mathrm{RV}}<\mathrm{PA}_{\mathrm{T}}<\mathrm{PA}_{\mathrm{CO}}$ while $\mathrm{PA}_{\mathrm{RV}}<\mathrm{PA}_{\mathrm{CO}}$ 


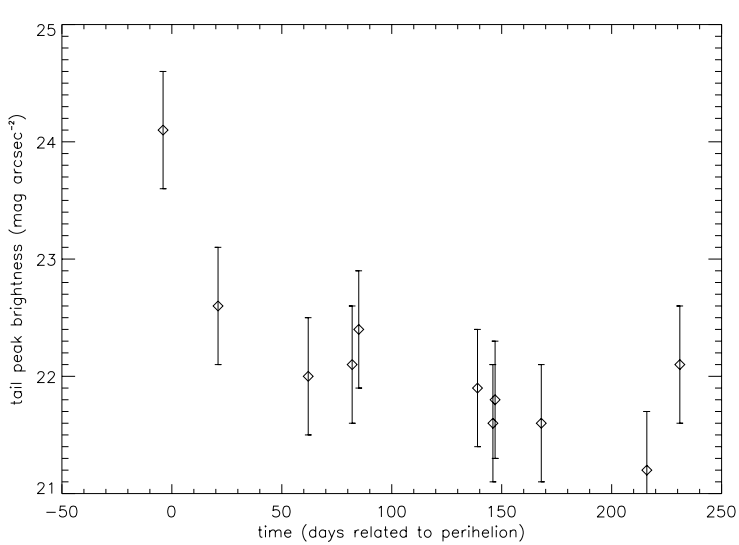

Fig. 2. Peak surface brightness of the thin tail observed during 2002 and 2003. Before perihelion, the much fainter tail is probably an ion tail. For $t-T>50$ days the tail brightness remains fairly constant, suggesting that the dust tail is characterized by a constant dust cross section. The magnitudes were corrected for the changing Sun-Comet distance $r$. See Table 1 for the relationship among $t-T$, UT and $r$.

and $\mathrm{PA}_{\mathrm{CO}}<\mathrm{PA}_{\mathrm{T}}<\mathrm{PA}_{\mathrm{RV}}$ while $\mathrm{PA}_{\mathrm{CO}}<\mathrm{PA}_{\mathrm{RV}}$, then the tail can be a dust tail. The data in Table 1 show that this interpretation is consistent with all available observations. After February 2003, the $\mathrm{PA}_{\mathrm{T}}$ and $\mathrm{PA}_{\mathrm{RV}}$ data show that the thin tail was apparently pointing towards the Sun. However, the condition $\mathrm{PA}_{\mathrm{RV}}<\mathrm{PA}_{\mathrm{T}}<\mathrm{PA}_{\mathrm{CO}}$ was always satisfied, so that in $3 \mathrm{D}$ space the thin tail was always placed outside the comet orbit. In other words, after February 2003 the tail was simply a perspective antitail, but we will continue to call it thin tail.

Since October 2002, all CCD images show a tail with almost perfectly constant shape and brightness (Table 1). Figure 2 shows the peak mag $\operatorname{arcsec}^{-2}$ of the thin tail between August 2002 and April 2003, after we have subtracted $5 \log r$ to correct for the changing Sun-Comet distance $r$ (the surface brightness does not depend upon the changing Earth-Comet distance). For $t-T>50$ days, the tail brightness remains fairly constant, suggesting that the total dust cross section remained constant (within a factor 3 ) since October 2002. Around perihelion the tail is much fainter: it could be either an ion tail or a dust tail composed of small grains ejected at perihelion only, much fainter than the dust tail appearing after perihelion.

\section{Neck-Line models}

Kimura \& Liu (1977) were the first to point out that on long time scales the heliocentric motion of the dust particles building up cometary dust tails has strong consequences on all observable structures belonging to dust tails: Neck-Lines are among the most prominent ones when the Earth is close to the comet orbital plane, and this condition was always satisfied during 2002 and 2003 thanks to the very low inclination $\left(7^{\circ}\right)$ of the orbital plane of $67 \mathrm{P}$ with respect to the ecliptic. To understand Neck-Lines it is necessary to introduce the concept of dust shells ejected by the inner coma of the comet. Let us consider dust of a single $\beta$ ejected at a single time from the comet nucleus. Here:

$\beta=\frac{C_{\mathrm{pr}} Q_{\mathrm{pr}}}{\rho_{\mathrm{d}} d}$ is one of the two parameters (the other is the starting velocity of the dust) determining the orbit of the grain, $C_{\mathrm{pr}}=$ $1.19 \times 10^{-3} \mathrm{~kg} \mathrm{~m}^{-3}$ depends on the light velocity, Solar mass and gravitation constant, $Q_{\mathrm{pr}}$ depends on the chemical composition of the grains and is close to one for the presumably large absorbing grains in the 67P tail (Burns et al. 1979; the fact that the $67 \mathrm{P}$ tail is composed by $\mathrm{mm}$-sized grains is confirmed by all models), $\rho_{\mathrm{d}}$ is the dust bulk density and $d$ is the dust diameter if grains are spheres, or the mean size if grains are aspherical but compact (i.e. far from fractal or strongly elongated shapes).

If we consider dust grains ejected in all directions at the same time with the same $\beta$ and with the same velocity, we obtain a spherical shell expanding in space. In a cometocentric reference frame, this shell is expected at first sight to maintain its spherical shape for all future times, but this naive conclusion is wrong, because it does not take into account that the cometocentric reference frame is not inertial. If, in the inertial heliocentric reference frame, we take into account the heliocentric keplerian orbit of each grain composing the spherical shell, we can compare dust orbits to the comet orbit: these two orbital planes intersect one another along a nodal line crossing the Sun, with the first node at the dust ejection and the second node again crossing the orbit plane of the comet nucleus at a true anomaly exactly $180^{\circ}$ after the ejection. The shell center motion is that of a particle with the same $\beta$ ejected at zero velocity, so that its positions are given by classical syndynesynchrone computations: it moves in the comet orbital plane. Monte Carlo simulations (Fulle \& Sedmak 1988) have shown that, when the shell center reaches a true anomaly exactly $180^{\circ}$ after the ejection, the shell can be well approximated by an ellipsoid (Fig. 3), whose two main axes in the comet orbital plane are approximately given by the ejection velocity times the flight time, and are orders of magnitude longer than the axis perpendicular to the comet orbital plane: we can approximate such an ellipsoid with a 2D ellipse shrunk on the same plane. If the observer is close to this plane, all the dust is confined in an infinitesimally thin sky area, forcing the shell brightness to rise to very high values.

If we consider shells of many $\beta$ values, we obtain a long spike starting from the comet nucleus which is much brighter than the surrounding tail and is named Neck-Line. In case of anisotropies of dust ejection and velocity, due both to nucleus inhomogeneity and asphericity and to the dust-gas interaction in the coma, the shells acquire a 3D shape more complex than ellipsoids, but always these 3D shells are well approximated by 2D figures (more complex than ellipses) at the second orbital node. In fact, Monte Carlo simulations have confirmed that the singularity generated by the dust shell collapse implies a brightness maximum well observable for any ejection anisotropy, and continuous temporal evolution of the dust loss rate and size distribution (Cremonese \& Fulle 1989). Fulle (1988) has also provided an analytical proof that the Neck-Line brightness must rise to infinity when the observer crosses the comet orbital plane. In such a limiting case the opacity of the dust grains comes into play and limits the brightness to a finite value. Neck-Lines were observed in many comets: Arend-Roland (Kimura \& Liu 1977), Bennett (Pansecchi et al. 1987; Fulle \& Sedmak 1988), Halley 

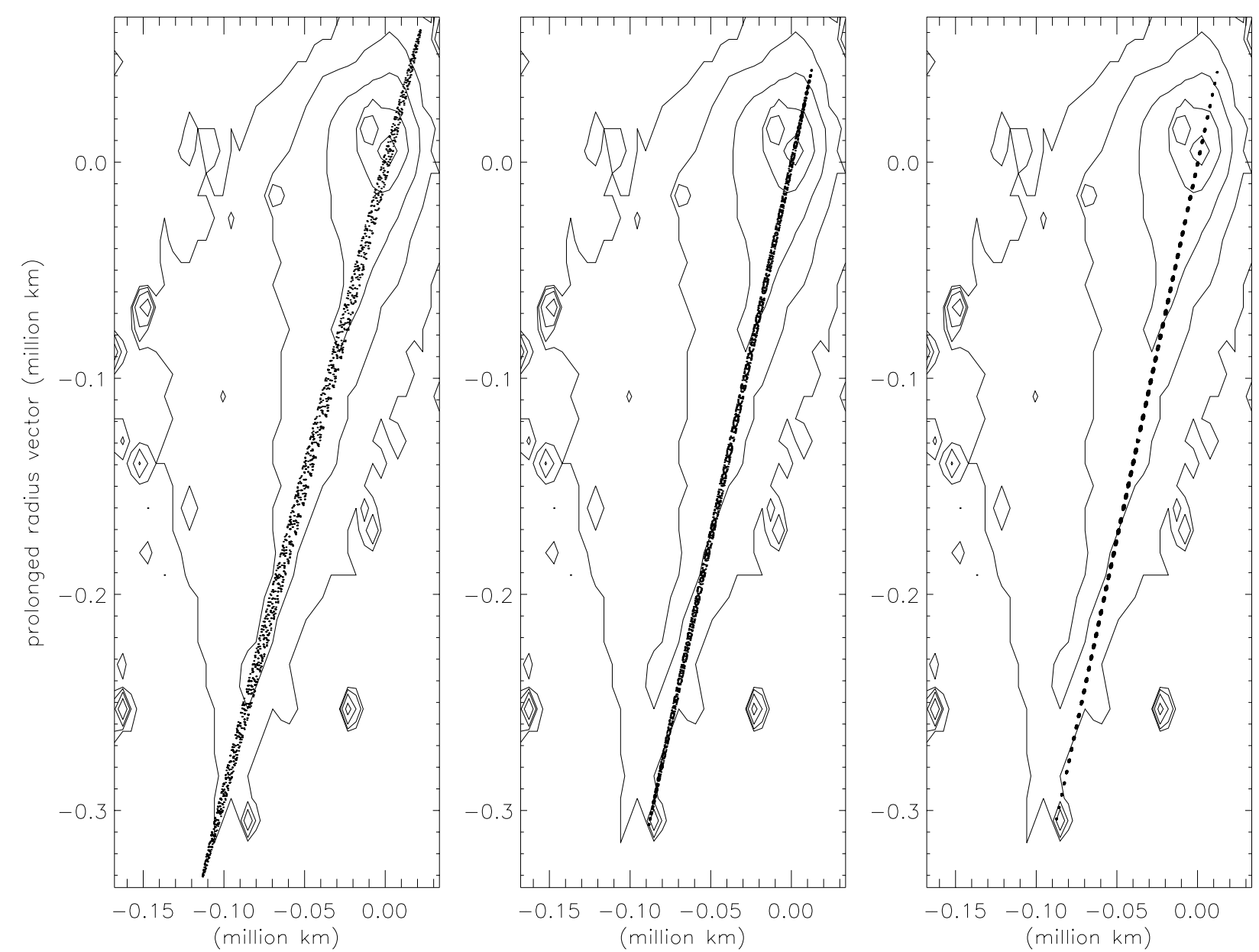

Fig. 3. Comparison among three different dust shells composed of dust of fixed $\beta$ and ejected at fixed times with fixed ejection velocities. The shells are followed in their heliocentric orbits and then projected on the sky at the TNG observation (see Table 1 for the observation log; at this time the thin tail appeared as a perspective antitail; a factor two intensity step was chosen between isophotes). The prolonged radius vector is the vector $r$ (Table 1) crossing the comet nucleus and prolonged outside the comet orbit, so that in all the panels the Sun is at image bottom. The comet nucleus position is exactly at $x=y=0$ (the brightness peak to the upper left of the nucleus is a star). Left panel: shell composed of grains of $\beta=1.5 \times 10^{-4}$ ejected at $3 \mathrm{~m} \mathrm{~s}^{-1}$ at 105.8 days before 2002 perihelion. Central panel: shell composed of grains of $\beta=5 \times 10^{-6}$ ejected at $0.12 \mathrm{~m} \mathrm{~s}^{-1}$ at the 1996 perihelion. Right panel: shell composed of grains of $\beta=2.5 \times 10^{-6}$ ejected at $0.06 \mathrm{~m} \mathrm{~s}^{-1}$ at the 1989 perihelion. The sample clustering is due to the $10^{-6}$ ratio between dust and comet nucleus orbital velocity.

(Cremonese \& Fulle 1989), Austin (Fulle et al. 1993) and others (e.g., Hale-Bopp at the beginning of 1998). It is probable that all the perspective months-old antitails observed in the past (e.g., Sekanina \& Schuster 1978) were in fact Neck-Lines. Neck-Lines ejected after perihelion could be observed before the following perihelion of periodic comets. However, the time between ejection and observation is so long (close to the comet orbital period) that the dust shells become long strings (Fig. 3), whose sky surface density is independent of the observation geometry.

In Table 1 we report the computed Position Angle $\mathrm{PA}_{\mathrm{NL}}$ of the 67P Neck-Line, which changes by only $5^{\circ}$ during the whole of 2003, while the antisolar direction $\mathrm{PA}_{\mathrm{RV}}$ changes by about $180^{\circ}$. Therefore, the apparent direction of the Neck-Line changes from an anti-sunward to a sunward spike. After October 2002, all the computed $\mathrm{PA}_{\mathrm{NL}}$ values fit the observed $\mathrm{PA}_{\mathrm{T}}$ values within the measurement errors. Moreover, a Neck-Line is expected after February 2003, appearing as a very thin perspective spike-like antitail. We can conclude that since October 2002 the observed tail was a Neck-Line, composed of dust ejected at times $t(\theta-\pi)$ and at Sun-Comet distances $r(\theta-\pi)$, different for each observation. The Neck-Line maintained an almost constant brightness since October 2002 (Fig. 2): this implies that the total cross section of the ejected dust was constant (within a factor 3) from 3.6 AU to $1.7 \mathrm{AU}$ before perihelion (see the $r(\theta-\pi)$ values in Table 1).

\section{Dust from previous perihelion passages}

At observation wavelengths of $12 \mu \mathrm{m}, 25 \mu \mathrm{m}$ and $60 \mu \mathrm{m}$, the IRAS satellite (Sykes \& Walker 1992) detected a trail of 67P, $1.1^{\circ}$ long behind the coma, and $0.1^{\circ}$ ahead the coma. The trail length allowed Sykes \& Walker (1992) to conclude that the whole 67P trail was ejected during the previous perihelion passage. Reach et al. (2003) suggested that dust released at the previous perihelion (January 1996) could have built up the thin tail of Fig. 1, which in this case should be identified with the IRAS trail (in fact, a trail can be defined as the dust tail ejected 
during previous perihelion passages). Then, the presumably large activity at the 1996 perihelion could explain the appearance of a bright dust trail during 2002 and 2003. To check this hypothesis, we use again the concept of dust shells: we consider a fixed time of dust ejection, a fixed $\beta$, and consider dust ejected in all the possible directions with a fixed velocity.

Here we compare the Neck-Line and the trail interpretations, by projecting on the sky dust shells exactly at the time of the TNG observation (Table 1): the comparison between the TNG CCD image (Fig. 3) and the sky-projected shells will allow us to check the reliability of the hypothesis. The shell ejection times are fixed: 1989 perihelion, 1996 perihelion and 105.8 days before the August 2002 perihelion (this last time is provided by $t(\theta-\pi)-T$ in Table 1 for the TNG observation). The $\beta$ and ejection velocity values are constrained by this fact: the TNG image shows that the brightness of the thin tail decreases by a factor 10 at least from the coma up to $3 \times 10^{5} \mathrm{~km}$ sunward (Fig. 3): therefore, the dust shell cannot be larger than the size of Fig. 3, otherwise we cannot fit such a fast brightness drop.

To compute the dust motion lasting up to 15 years, we neglect planetary perturbations. In fact, we consider very large grains, orbiting very close to the comet nucleus: it is probable that planetary perturbations in the course of the 15 years affect the grains in exactly the same way they affect the comet nucleus. Moreover, the orbital parameters of 67P have remained almost constant since 1989: the three angles describing the comet orbit orientation changed by less than $0.1^{\circ}$, while the perihelion distance and eccentricity changed by less than $1 \%$ (Yeomans \& Wimberly 1991). Since we are considering grains orbiting very close to the nucleus, the differences between the orbital angles of grains and of nucleus will be much lower than $0.1^{\circ}$ and the difference between perihelion distance and eccentricity of each grain and of the nucleus will be lower than $1 \%$.

The left panel of Fig. 3 shows the sky-projected dust shell composed of grains with $\beta=1.5 \times 10^{-4}$ (corresponding to a diameter of $0.8 \mathrm{~cm}$ if $\rho_{\mathrm{d}}=10^{3} \mathrm{~kg} \mathrm{~m}^{-3}$ ) ejected at $3 \mathrm{~m} \mathrm{~s}^{-1}$ at 105.8 days before 2002 perihelion. This is a Neck-Line shell perfectly fitting the Position Angle of the observed thin tail. The central panel of Fig. 3 shows the sky-projected dust shell composed of grains of $\beta=5 \times 10^{-6}$ (corresponding to a diameter of $24 \mathrm{~cm}$ if $\rho_{\mathrm{d}}=10^{3} \mathrm{~kg} \mathrm{~m}^{-3}$ ) ejected at $0.12 \mathrm{~m} \mathrm{~s}^{-1}$ exactly at the 1996 67P perihelion. The right panel of Fig. 3 shows the sky-projected dust shell composed of grains of $\beta=2.5 \times 10^{-6}$ (corresponding to a diameter of $48 \mathrm{~cm}$ if $\rho_{\mathrm{d}}=10^{3} \mathrm{~kg} \mathrm{~m}^{-3}$ ) ejected at $0.06 \mathrm{~m} \mathrm{~s}^{-1}$ at the $198967 \mathrm{P}$ perihelion. These last two shells do not fit the Position Angle of the observed thin tail: the difference is larger than $3^{\circ}$, much larger than the small uncertainties introduced by planetary perturbations occurring during 15 years. The preceding dust velocities are much lower than the expected escape velocity $v_{\text {esc }}$ from the 67P nucleus. Assuming a nucleus radius $R_{\mathrm{n}}=2 \mathrm{~km}$ (Lamy et al. 2003), we get

$v_{\text {esc }}=0.75 \sqrt{\frac{\rho_{\mathrm{n}}}{10^{3} \mathrm{~kg} \mathrm{~m}^{-3}}} \frac{R_{\mathrm{n}}}{1 \mathrm{~km}} \mathrm{~m} \mathrm{~s}^{-1}>0.5 \mathrm{~m} \mathrm{~s}^{-1}$

for reasonable nucleus densities $\rho_{\mathrm{n}}$. Since the gas coma is probably inhomogeneous, most of the meter-sized boulders leaving the nucleus surface will fall back on the nucleus, while a negligible fraction only will leave the coma with $v \ll v_{\text {esc }}$ (Crifo et al. 2004). To obtain an observable fraction of such boulders, their loss rate at the 1996 or 1989 perihelia should have been so large as to be inconsistent with the observed coma brightness. Models based on extremely anisotropic ejections at previous perihelion passages cannot solve the problem either. To obtain the shells plotted in Fig. 3 with much larger $\beta$ values, all the dust must have been ejected in sectors covering infinitesimal fractions of the solid angle: realistic physical dust-gas interaction can neither reproduce such a finely collimated lonesome dust jet, nor change the direction of such a jet to fit the tail orientation at all observation times.

We must conclude that the thin tail shown in Fig. 3 is not a trail. This means that the 67P IRAS trail must be fainter than the observed thin tail. The IRAS trail was wider $\left(5 \times 10^{4} \mathrm{~km}\right)$ than the thin tail shown in Fig. 3. Sykes \& Walker (1992) derive an optical depth $\tau=2 \times 10^{-9}$ of dust at the brightness peak of the trail, so that we can compute the corresponding peak optical brightness if we assume that the dust area emitting at IR wavelengths is the same area that is scattering the sun light: this is correct because the dust sizes in the trail are certainly much larger than both optical and IR wavelengths. Then

$$
m_{\text {trail }}=m_{\odot}+2.5 \log \frac{\pi(\delta r)^{2}}{A_{\mathrm{p}} \tau}
$$

where $m_{\text {trail }}$ is the trail peak mag $\operatorname{arcsec}^{-2}$ in the same passband as the Solar magnitude $m_{\odot}, \delta=206265 \operatorname{arcsec}, r$ is the Sun-Comet distance in $\mathrm{AU}, A_{\mathrm{p}}$ is the albedo, set here to $4 \%$. We obtain that the IRAS trail is fainter than 28.0 mag $\operatorname{arcsec}^{-2}$ in the $R$ passband. This proves that the IRAS trail is much fainter than the thin tail observed during 2002 and 2003 (Fig. 2), confirming the conclusions already reached considering the disagreement between $\mathrm{PA}_{\mathrm{T}}$ and $\mathrm{PA}_{\mathrm{CO}}$ in Table 1 . On the other hand, since the Neck-Line is much brighter than the trail, IRAS must have observed it. However, the poor angular resolution (the IRAS cameras had pixels 2 arcmin wide, equivalent to 100 pixels in Fig. 1) prevented IRAS from disentangling the two dust structures in the sky: the Neck-Line remained confused in the first 5 pixels of the trail observed by IRAS.

\section{Neck-Line photometry}

The images showing the longest Neck-Line of 67P were taken at the Tautenburg Schmidt Telescope during the nights of 27 and 28 March, 2003 (Fig. 1, Weiler et al. 2004a). The CCD field of this telescope is much wider than that of the TNG Telescope. Therefore, the Neck-Line data from these images allow us to explore the dust population at the smallest sizes we are able to detect (the farther the dust from the nucleus, the smaller the grains in the Neck-Line). We take into account only $R$-filtered images to increase the $\mathrm{S} / \mathrm{N}$ ratio. In Table 2 we describe the geometry of the Tautenburg observations and the angles defining the expected orientation of the Neck-Line: the observed Position Angle of the Neck-Line $\mathrm{PA}_{\mathrm{T}}$ coincides with the computed $\mathrm{PA}_{\mathrm{NL}}$ within the measurement accuracy (Table 1).

By means of simple analytical formulae, the Neck-Line model allows us to obtain both the $\beta$-dependence of the dust 
Table 2. Geometry of Neck-Line Observations. Time: March 2003. $r$ and $\Delta$ : Sun-Comet and Earth-Comet distances at observation. $\lambda$ : Earth cometocentric latitude on the comet orbital plane. $\Phi$ : phase angle. $\mathrm{PA}_{\mathrm{RV}}$ : Position Angle of the antisolar direction. $s, a$ and $b$ : geometric parameters of the Neck-Line (see Eq. (5)). $\alpha_{\mu}+\alpha_{\tau}$ : predicted Angle between the antisolar direction and the Neck-Line axis. $t_{\text {ejection}}$ : time of ejection of the dust composing the Neck-Line (days related to comet perihelion).

\begin{tabular}{ccccccccccc}
\hline \hline Time [UT] & $r[\mathrm{AU}]$ & $\Delta[\mathrm{AU}]$ & $\lambda\left[^{\circ}\right]$ & $\Phi\left[^{\circ}\right]$ & $\mathrm{PA}_{\mathrm{RV}}\left[{ }^{\circ}\right]$ & $s\left[10^{11} \mathrm{~m}\right]$ & $a\left[10^{-8} \mathrm{~s}^{-1}\right]$ & $b\left[10^{-6} \mathrm{~s}^{-1}\right]$ & $\alpha_{\mu}+\alpha_{\tau}\left[^{\circ}\right]$ & $t_{\text {ejection }}[\mathrm{days}]$ \\
\hline 27.875 & 2.621 & 1.694 & 2.9 & 10.1 & 132.19 & 9.50 & 1.447 & 0.978 & 162.14 & -105.37 \\
28.028 & 2.622 & 1.696 & 2.9 & 10.1 & 132.03 & 9.50 & 1.447 & 0.981 & 162.29 & -105.30 \\
28.896 & 2.628 & 1.707 & 2.8 & 10.4 & 131.14 & 9.50 & 1.449 & 0.993 & 163.15 & -104.90 \\
\hline
\end{tabular}

velocity (and its absolute values in $\mathrm{m} \mathrm{s}^{-1}$ for each $\beta$ value), and the $\beta$-distribution, which is related to the dust size distribution. If we assume a constant dust bulk density in the relatively small $\beta$ interval actually covered by the Neck-Line (a factor 6 only between the largest and smallest $\beta$ values), we immediately obtain the $u$ value (power index of the dust ejection velocity versus the dust radius $\left.s, v=v_{0}\left(s / s_{0}\right)^{u}\right)$, and the power index $\alpha$ of the differential dust size distribution. In fact, Fulle $\&$ Sedmak (1988) have developed the photometric theory of the Neck-Lines and obtain that, if this condition is satisfied:

$v(\beta)<\beta$ s $a$

then the sky surface brightness of the Neck-Line is

$D(x, y)=\frac{F(t, \beta)}{s v(\beta)}\left[1+\operatorname{erf} \frac{a x}{v(\beta)}\right] \exp \left[-\frac{b^{2} y^{2}}{v^{2}(\beta)}\right]$

where $F$ is the $\beta$ distribution, $s, a$ and $b$ are geometrical parameters of the Neck-Line (listed in Table 2 for the Tautenburg observations), $v(\beta)$ is the dust ejection velocity, and $x$ and $y$ define an image reference frame oriented as follows: $x$ lies exactly along the Neck-Line axis, while $y$ is exactly perpendicular to the Neck-Line axis. Equation (5) shows that, when we measure the half-width of the Neck-Line along the $y$ direction, we directly measure the dust velocities at given $\beta$ values: only this method is able to provide such direct information from ground based observations. Then, the brightness measured along the Neck-Line allows us to compute the $F$ values. If we fit $F$ by means of a power law with index $\gamma$, we get the power index $\alpha=-\gamma-4$ of the corresponding differential dust size distribution at the nucleus.

Figure 4 shows the results regarding the dust ejection velocity. From Table 2 we obtain that all the data from all the three Tautenburg observations provide information on the dust ejection that occurred during 10 hours only at $t-T=-105$ days. We can consider constant the mean dust environment of the comet during these $10 \mathrm{~h}$, so that we can refer all data to a single dust velocity function. This is important because we can extract few data from each image: many star tracks pollute the photometric data of the Neck-Line, and since these tracks differ in orientation by less than $30^{\circ}$ with respect to the $x$-axis (Fig. 1), every directional filtering removing the stars would affect the width of the Neck-Line we need to measure. We prefer to select in each image the Neck-Line sector which is not polluted by any track. This reduces the number of useful sectors in each data set. Collecting together all the results, we improve the sampling of the $F$ and $v$ functions. In total we were able

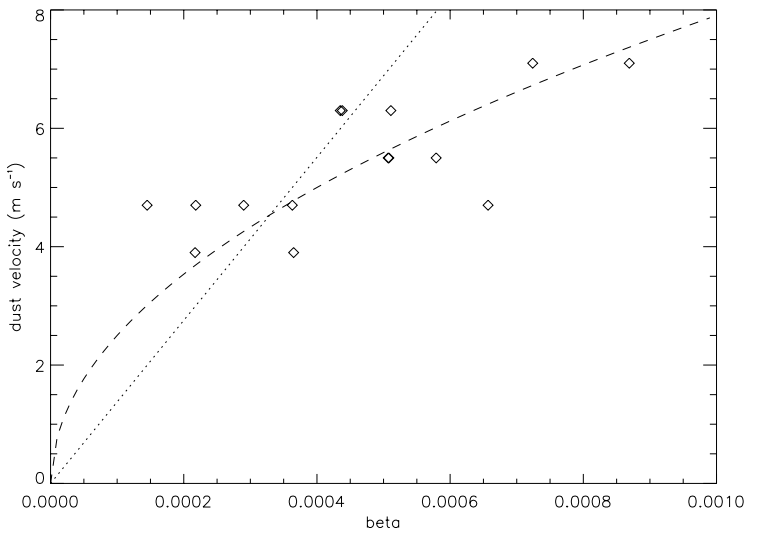

Fig. 4. Dust velocity function provided by the Neck-Line model applied to Tautenburg Schmidt CCD images. Diamonds: width of the Neck-Line (converted to dust velocity $v=b \times$ width) at several $x$ axis positions (converted to $\beta=x / s, b$ and $s$ in Table 2). Dotted line: threshold of validity of the analytical approximations (Eq. (4)). Dashed line: $\sqrt{\beta}$ curve, corresponding to $u=-1 / 2$ in Figs. 6 and 7 .

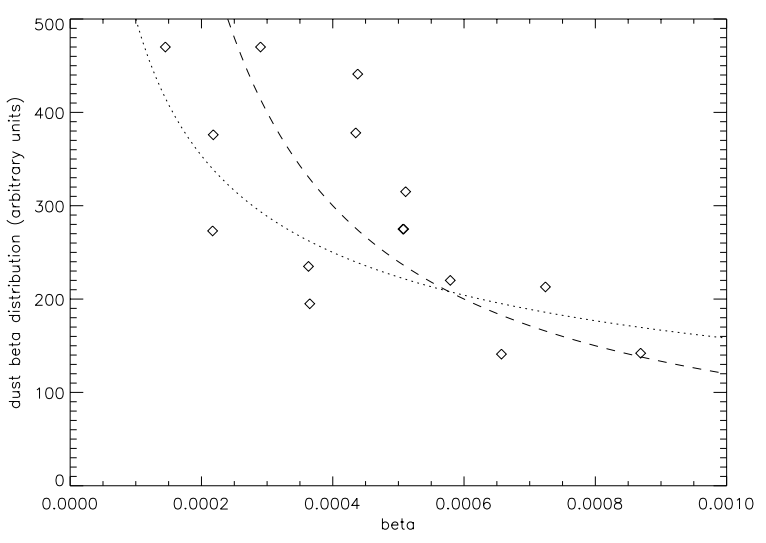

Fig. 5. $\beta$ distributions obtained by the photometric theory of Neck-Lines applied to the same Tautenburg Schmidt images already analysed in Fig. 4. Diamonds: $\beta$-distribution $F$ obtained by means of Eq. (5) (see text for discussion). Dotted line: $\beta$ distribution with power index $\gamma=-0.5$, corresponding to $\alpha=-3.5$. Dashed line: $\beta$ distribution with power index $\gamma=-1$, corresponding to $\alpha=-3$.

to measure 14 width and brightness values of the Neck-Line. The widths are already converted into velocities in Fig. 4 by means of the $b$ parameter (Table 2). The dashed line is the best $\beta$ power law fitting the points, yielding $u=-1 / 2$.

Figure 5 shows the results regarding the $\beta$ distribution, i.e. the brightness values along the $x$-axis converted into $F$ by means of Eq. (5). The $F$ values can be fitted by any power 

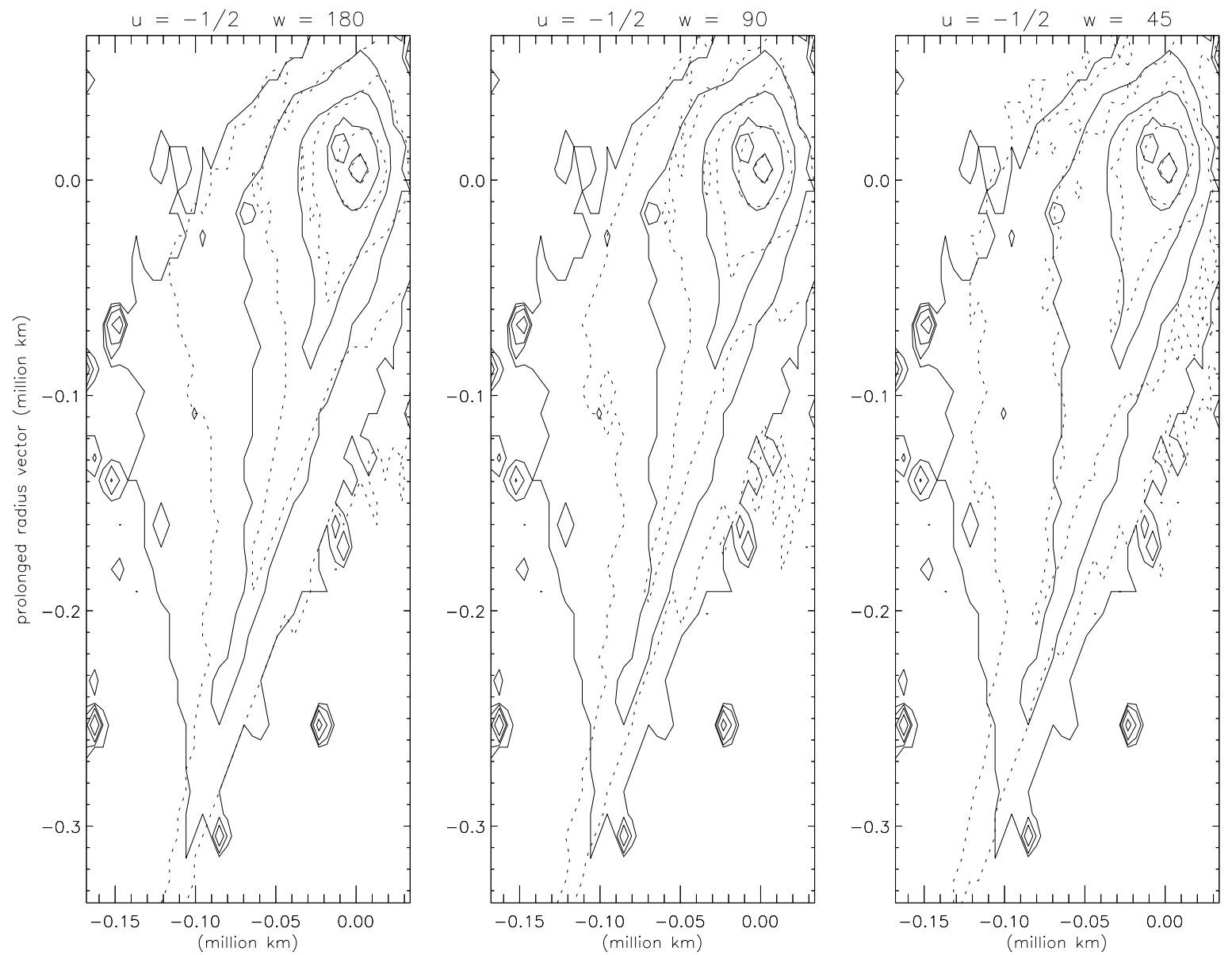

Fig. 6. Image of the tail of comet $67 \mathrm{P}$ obtained on 27.0 March 2003 by means of the TNG Galileo Telescope at La Palma. In all the panels the Sun is at the bottom. A factor two intensity step was chosen between isophotes. Continuous lines: isophotes of the observed image. Dashed lines: isophotes of the model tail computed by means of the inverse tail model adopting the dust parameters summarized in Fig. 7. The panels refer to different combinations of the $w$ and $u$ parameters. $w$ refers to the anisotropy of dust ejection: $w=180^{\circ}$ for perfectly isotropic dust ejection from the inner coma; $w=90^{\circ}$ for dust ejection confined to the half coma facing the Sun; $w=45^{\circ}$ for dust ejection confined within a cone with its axis pointing to the Sun and with a half width of $45^{\circ} . u$ models the dependence of the dust ejection velocity $v$ on the dust radius $s$ : $v=v_{0}\left(s / s_{0}\right)^{u}\left(v_{0}\right.$ and $s_{0}$ are shown in Fig. 7). The value $u=-1 / 2$ obtained in Fig. 4 was adopted.

index $\gamma$ between -0.5 and -1 , implying $-3.5 \leq \alpha \leq-3.0$. This result refers to the $\beta$ range shown in Figs. 4 and 5, corresponding to dust sizes from $1.5 \mathrm{~mm}$ to $1 \mathrm{~cm}$ if we consider valid Eq. (1) with $\rho_{\mathrm{d}}=10^{3} \mathrm{~kg} \mathrm{~m}^{-3}$. The fact that $\alpha \geq-3.5$ would be further confirmed if we took into account the fact that at the smallest $\beta$ values the condition given by Eq. (4) is not satisfied. In such a case, a numerical integral of the NeckLine brightness should be adopted instead of Eq. (5), which always provides $F$ distributions characterized by lower $\gamma$ values, implying higher $\alpha$ values. Therefore, we can conclude that the dust population of 67P ejected at 105 days before perihelion is dominated both in mass and in brightness by the largest (mm-sized) ejected grains. We can extrapolate this conclusion for all the dust ejected from 3.6 AU to 1.7 AU before perihelion, because the Neck-Line has maintained the same shape and peak brightness since October 2002 (Fig. 2).

\section{Dust tail fit by means of the inverse model}

The tail images with the best $\mathrm{S} / \mathrm{N}$ ratio were obtained when comet $67 \mathrm{P}$ was observed as target of opportunity by means of the Galileo Telescope (TNG) at La Palma on the night between 26 and 27 March, 2003. We selected all the obtained R CCD images and added them to obtain an input image to be processed by means of the inverse tail model (Fulle 1989). Isophotes of the tail image are shown in Figs. 3 and 6, oriented such that the Sun is exactly in the $-y$ direction. The image is not polluted by any significant ion tail (which should lie close to the $+y$ direction) and is dominated by the Neck-Line discussed in the previous sections, which in this period appeared as a perspective dust antitail. In this section we use the inverse tail model to link the dust in the Neck-Line to the dust in the surrounding tail and the coma close to the nucleus. In fact, the inverse dust tail model reconstructs a synthetic tail built up by dust ejected during a finite time interval. If we choose this time interval large enough to cover both the ejection of Neck-Line dust (about one year before the observations) and the coma dust (a few days before the observations), we can link together the dust parameters during the whole of the 67P activity. The tail model provides dust parameters to be compared with the same quantities obtained by means of the independent Neck-Line 

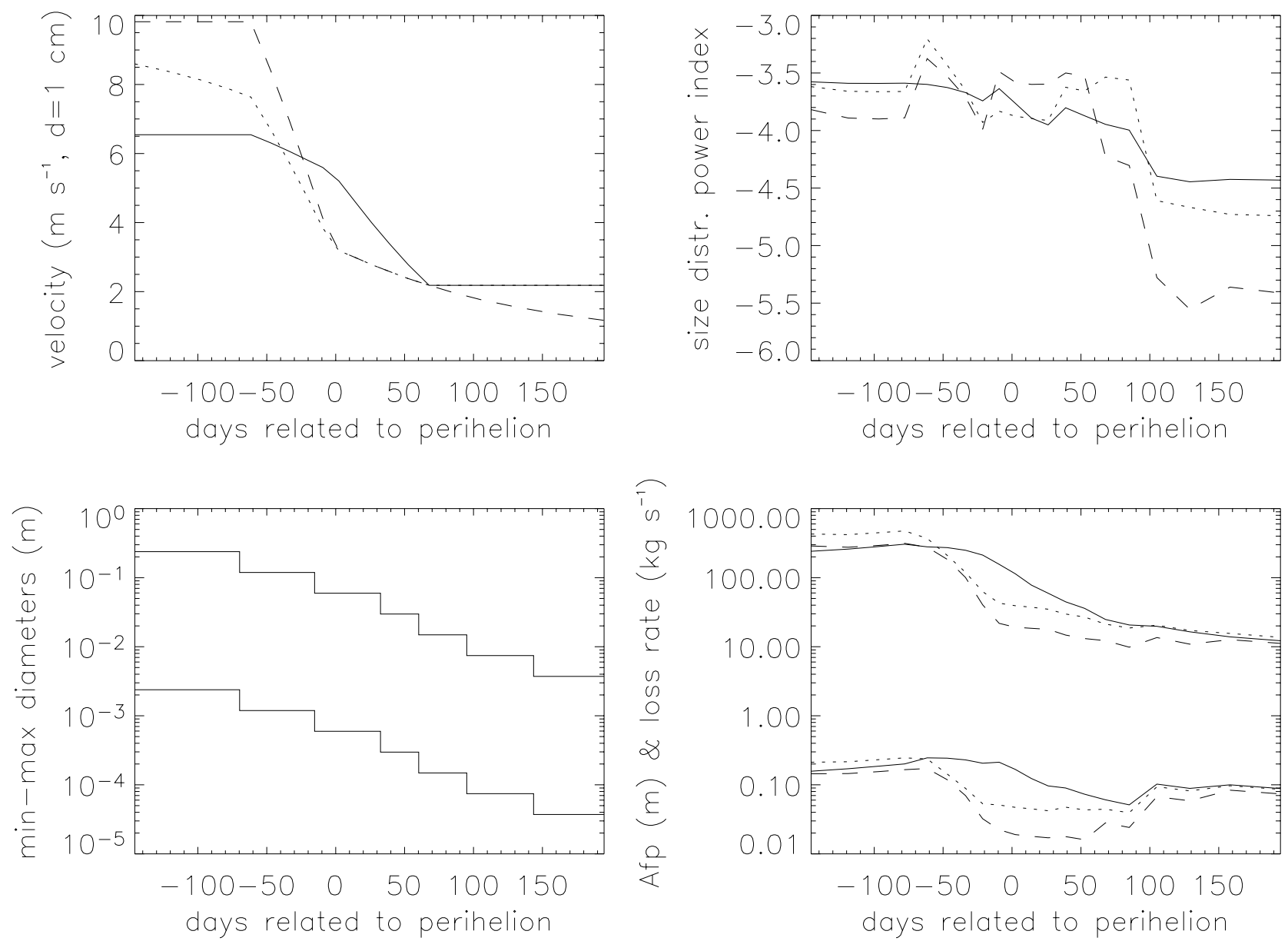

Fig. 7. Dust parameters describing the dust environment of 67P during the 2002 perihelion passage. The curves are related to the combinations of the parameters $w$ and $u$ described in Fig. 6. Continuous lines: $w=180^{\circ}$ and $u=-1 / 2$. Dotted lines: $w=90^{\circ}$ and $u=-1 / 2$. Dashed lines: $w=45^{\circ}$ and $u=-1 / 2$. Upper-left panel: dust ejection velocity from the inner coma for a reference radius $s_{0}=5 \mathrm{~mm}$. Lower-left panel: interval of the dust diameters to which all the shown outputs are related. Upper-right panel: power index of the differential size distribution. Lower-right panel: dust mass loss rate (upper curves, $\mathrm{kg} \mathrm{s}^{-1}$ ) and $A f \rho_{\mathrm{c}}$ values (lower curves, $\mathrm{m}$ ) of the dust grains inside the size interval shown at left. All curves show an asymmetry between pre and post-perihelion times. See Table 1 for the relationship among $t-T$, UT and $r$.

photometry, thus testing the reliability of the outputs of both models. The input TNG images were not calibrated by means of standard stars, so that the isophote levels shown in Fig. 6 refer to arbitrary brightness levels. However, the dust tail model also provides the expected $A f \rho_{\mathrm{c}}$ values (A'Hearn et al. 1984):

$A f \rho_{\mathrm{c}}=2 \pi A_{\mathrm{p}}(\Phi) Q_{\mathrm{d}} \int_{s_{1}}^{s_{2}} \frac{s^{2} g(s)}{v(s)} \mathrm{d} s$

to be compared with the observed $A f \rho_{\mathrm{o}}$ values obtained by means of coma absolute photometry. In Eq. (6), $A_{\mathrm{p}}(\Phi)$ is the dust albedo times the phase function, $s_{1}$ and $s_{2}$ are the smallest and largest dust radii considered by the model, $Q_{\mathrm{d}}$ is the cumulative dust number loss rate between $s_{1}$ and $s_{2}, g(s)$ is the dust size distribution and $v(s)$ the dust velocity. Therefore, the comparison between $A f \rho_{\mathrm{o}}$ and $A f \rho_{\mathrm{c}}$ allows us to calibrate in absolute units (meters) the computed $A f \rho_{\mathrm{c}}$ values provided by the inverse tail model. Since the relationship between $A f \rho_{\mathrm{c}}$ and the mass loss rate computed by the inverse tail model depends on the dust albedo only, by making the usual assumption that $A_{\mathrm{p}}(\Phi)=0.04$ we can calibrate in absolute units $\left(\mathrm{kg} \mathrm{s}^{-1}\right)$ the dust loss rate provided by the tail model during all the covered times (including the Neck-Line ejection). The $A f \rho_{\mathrm{c}}$ values allow us also to calibrate absolutely in magnitudes the isophotes shown in Figs. 3 and 6: we obtain that the faintest isophote in Figs. 3 and 6 is $26.6 \pm 0.3 \mathrm{mag} \mathrm{arcsec}^{-2}$ in the Cousins $R$ passband, thus confirming that we were unable to detect the IRAS trail by means of the deepest performed CCD observations.

The inverse tail model consists in a least squares fit of the surface intensity of the dust tail sampled in 1600 pixels. The output of such a fit is a set of dust parameters that allows us to minimize the error between the model image and the input image (Fulle 1989). The fits of the observed tail shown in Fig. 6 depend on two parameters describing the dust ejection from the inner coma, $u$ (defined in the previous section) and $w$, which describes the anisotropy of dust ejection from the inner coma. Usually, all the $u$ and $w$ combinations provide fits with very similar errors, so that inverse tail models are unable to disentangle the best $u$ and $w$ combination. Here also, as usual, the errors of the fits shown in Fig. 6 are very similar (19\%, 19\% and $20 \%$ of the total brightness). But the 67P Neck-Line offers the rare opportunity to select a preferred $u$ and $w$ combination. 
The parameter $u=-1 / 2$ has been determined in the previous section by means of Neck-Line photometry, so that the dust velocity $v(s)$ can be described by

$v(s)=v_{0} \sqrt{s_{0} / s}$

becoming equal to $v_{0}$ at the dust radius $s_{0}$. The fits of the Neck-Line shown in Fig. 6 constrain the best $w$ value. Let us consider the axis of the Neck-Line at the second isophote level: its coordinates are $x=-9 \times 10^{4} \mathrm{~km}$ and $y=-2.5 \times 10^{5} \mathrm{~km}$. If we join this point to the comet nucleus by means of a straight line, we define the observed Neck-Line axis, which crosses the point at $x=-6 \times 10^{4} \mathrm{~km}$ and $y=-1.9 \times 10^{5} \mathrm{~km}$ precisely, i.e. the Neck-Line axis at the second isophote level computed by means of $w=180^{\circ}$. The corresponding points related to $w=90^{\circ}\left(x=-7 \times 10^{4} \mathrm{~km}\right.$ and $\left.y=-2.0 \times 10^{5} \mathrm{~km}\right)$ and $w=45^{\circ}\left(x=-8 \times 10^{4} \mathrm{~km}\right.$ and $\left.y=-2.1 \times 10^{5} \mathrm{~km}\right) \mathrm{do}$ not lie on the same observed Neck-Line axis: the stronger the anisotropy of dust ejection, the larger the difference in direction between the observed and computed Neck-Lines. Regarding the physical meaning of this result, we remember that physical models of dust ejection (Crifo \& Rodionov 1999; Crifo et al. 2004) provide dust flux ratios between the day and night side of the coma (not the nucleus) smaller than a factor 4 . In the schematic approximation adopted here, such a difference between day and night coma sides provides ejection much closer to the $w=180^{\circ}$ case rather than $w=90^{\circ}$.

Figure 7 shows the dust parameters provided by the inverse tail model. We show the outputs corresponding to all $u$ and $w$ combinations considered in Fig. 6, although the NeckLine fits suggest that only the continuous lines $(u=-1 / 2$ and $w=180^{\circ}$ ) should be taken into account. Fulle (2000) describes the complex procedure to obtain the time-dependent size interval (shown in the left-lower panel) inside which all the outputs are computed. The $A f \rho_{\mathrm{c}}$ values are certainly lower limits of the observed $A f \rho_{\mathrm{o}}$, which depend on all ejected dust sizes. Therefore, in order to recover the absolute units of $A f \rho_{\mathrm{c}}$ and mass loss rate, we set the highest $A f \rho_{\mathrm{c}}$ value at $t-T=$ 200 days, slightly lower than the $A f \rho_{\mathrm{o}}=0.4 \mathrm{~m}$ observed by Lamy et al. (2003) at $t-T=206$ days: the next section is devoted to a detailed analysis of the comparison between $A f \rho_{\mathrm{c}}$ and $A f \rho_{\mathrm{o}}$ values. Having recovered the absolute values (meters) of $A f \rho_{\mathrm{c}}$, it is possible to obtain the absolute mass loss rate by adopting $A_{\mathrm{p}}(\Phi)=0.04$. If the albedo is assumed to be $2 \%$, the mass loss rates become twice as large.

All the other output shows the same unusual asymmetry with respect to perihelion: larger values before than after. The velocity at radius $s_{0}=5 \mathrm{~mm}$ drops from about $7 \mathrm{~m} \mathrm{~s}^{-1}$ before perihelion to $2 \mathrm{~m} \mathrm{~s}^{-1}$ after. The power index $\alpha$ of the differential dust size distribution at the nucleus drops from $\alpha=-3.5$ before perihelion to $\alpha=-4.5$ after. This implies that the dust mass loss rate drops from about $200 \mathrm{~kg} \mathrm{~s}^{-1}$ before perihelion to about $10 \mathrm{~kg} \mathrm{~s}^{-1}$ after. The time-independent size distributions, loss rates and dust velocities obtained at $-150<t-T<-50$ days are consistent with the spike appearance of the Neck-Line, which in naive interpretations could suggest an outburst at $t-T=-105.8$ days: the tail brightness peak in the Neck-Line is uniquely due to the peak of sky-projected dust densities of the shells collapsed on the comet orbital plane.
To compare the results provided by Neck-Line photometry and inverse tail model, we must take into account that the results shown in Fig. 7 were converted from $\beta$ values to dust diameters by adopting Eq. (1) with a dust bulk density $\rho_{\mathrm{d}}=10^{3} \mathrm{~kg} \mathrm{~m}^{-3}$. In Fig. 7 we can adopt any other value of the dust bulk density: this will affect the dust diameter values only. For instance, if $\rho_{\mathrm{d}}=100 \mathrm{~kg} \mathrm{~m}^{-3}$, all the dust diameters should be multiplied by a factor ten, whereas the velocities, loss rates, $A f \rho_{\mathrm{c}}$ and $\alpha$ values will remain the same. At $s=5 \mathrm{~mm}$ and $t-T=-105$ days, Fig. 7 provides $v=6.5 \mathrm{~m} \mathrm{~s}^{-1}$ for the most probable $u=-1 / 2$ and $w=180^{\circ}$. Such a dust radius corresponds to $\beta=1.2 \times 10^{-4}$ for $\rho_{\mathrm{d}}=10^{3} \mathrm{~kg} \mathrm{~m}^{-3}$. At this $\beta$, we get from Fig. 4 a dust velocity close to $4 \mathrm{~m} \mathrm{~s}^{-1}$ : the difference is similar to the uncertainty affecting the obtained dust velocities. For $-150<t-T<-50$ days, the size range in Fig. 7 is wider than the $\beta$ range in Fig. 5, because larger dust sizes are taken into account, when we consider Eq. (1) with $\rho_{\mathrm{d}}=10^{3} \mathrm{~kg} \mathrm{~m}^{-3}$. The fact that in Fig. 5 we get $-3.0<\alpha<-3.5$ while in Fig. 7 we get $-3.5<\alpha<-3.8$ can be due either to a decrease of $\alpha$ at increasing sizes, or to uncertainties of the inverse tail model outputs. In the following, for $-150<t-T<-50$ days we will consider a mean value $\alpha=-3.4$.

\section{Comparison with dust coma photometry}

In this section we discuss the influence of the finite size interval inherent in the dust tail model (lower-left panel in Fig. 7) on the $A f \rho_{\mathrm{c}}$ and mass loss rates curves (lower-right panel in Fig. 7). This discussion for $A f \rho_{\mathrm{c}}$ is very important, because all the curves in the lower-right panel of Fig. 7 were calibrated by means of this quantity and because $A f \rho$ depends on all the quantities shown in Fig. 7. To evaluate how the finite size range influences $A f \rho_{\mathrm{c}}$, we did the following computations. We selected the most probable $u$ and $w$ parameter combination $\left(u=-1 / 2\right.$ and $w=180^{\circ}$ ). From the outputs in Fig. 7, we selected sample velocities and size distribution power indices, listed in Table 3. Then we assumed that the dust size distribution follows a power law with the same index $\alpha$ even outside the size interval covered by the model. Then we computed the number loss rates outside this range (Table 3 ) and the $A f \rho_{\mathrm{c}}$ obtained from these new loss rates. In this way we obtained how sensitive the $A f \rho_{\mathrm{c}}$ quantity is to these changes, and how much we can extrapolate the obtained dust parameters outside the size interval considered by the tail model. Then, if $g(s)$ is a power law with index $\alpha$, Eqs. (6), (7), and Fig. 7 yield

$Q_{\mathrm{d}}=\frac{\sqrt{s_{o}} v_{o}\left(s_{2}^{1+\alpha}-s_{1}^{1+\alpha}\right)(\alpha+3.5)}{2 \pi A_{\mathrm{p}}\left(s_{2}^{3.5+\alpha}-s_{1}^{3.5+\alpha}\right)(\alpha+1)} A f \rho_{\mathrm{c}}$

and then Eq. (6) with other values for $s_{1}$ provides $A f \rho_{\mathrm{c}}$ outside the size range shown in Fig. 7. The results are shown in Table 3, and show that the adopted extrapolations of the dust size range imply large changes of $A f \rho_{\mathrm{c}}$ around perihelion only: in particular, the rough minimum around perihelion in Fig. 7 becomes a well defined maximum at about 50 days after perihelion. The $A f \rho_{\mathrm{c}}$ values in Table 3 were computed adopting a dust radius of $1 \mathrm{~cm}$ as upper limit. Since certainly around perihelion much larger grains were ejected from the 67P coma, the $A f \rho_{\mathrm{c}}$ values 
Table 3. Dust parameters of 67P at several time intervals related to perihelion. $\alpha$ : power index of the differential dust size distribution (obtained from Fig. 7). $v_{0}$ : dust ejection velocity from the inner coma at the reference dust radius $s_{0}=5 \mathrm{~mm}$ (obtained from Fig. 7). $r$ : Sun-Comet distance. $A f \rho_{\mathrm{c}}$ : computed value over the extrapolated dust radius range between $1 \mathrm{~cm}$ and $0.5 \mu \mathrm{m} . N_{\text {lay }}$ : number of layers per day of grains larger than two microns deposited on a lander at the nucleus surface. $Q_{\mathrm{d}}\left[\mathrm{s}^{-1}\right]$ : cumulative dust number loss rate for all the grains with radius larger than $s$. No $Q_{\mathrm{d}}$ value is given when the extrapolation of the size distribution is not supported by any observation.

\begin{tabular}{|c|c|c|c|c|c|}
\hline$t-T$ [days] & $<-100$ & -50 & 0 & +50 & +150 \\
\hline$\alpha$ & -3.4 & -3.4 & -3.7 & -4.0 & -4.5 \\
\hline$v_{o}\left[\mathrm{~m} \mathrm{~s}^{-1}\right]$ & 6.5 & 6.0 & 5.5 & 4.0 & 3.0 \\
\hline$r[\mathrm{AU}]$ & $>1.7$ & 1.4 & 1.3 & 1.4 & 2.1 \\
\hline$A f \rho_{\mathrm{c}}[\mathrm{m}]$ & 0.1 & 0.2 & 1.0 & 1.7 & 0.5 \\
\hline$N_{\text {lay }}$ & 0.2 & 0.3 & 1.1 & 1.7 & 1.6 \\
\hline$Q_{\mathrm{d}}$ at $s>1 \mathrm{~cm}$ & $1.6 \times 10^{03}$ & $3.2 \times 10^{03}$ & $1.4 \times 10^{03}$ & $2.1 \times 10^{02}$ & $3.8 \times 10^{00}$ \\
\hline$Q_{\mathrm{d}}$ at $s>5 \mathrm{~mm}$ & $8.5 \times 10^{03}$ & $1.7 \times 10^{04}$ & $8.9 \times 10^{03}$ & $1.7 \times 10^{03}$ & $4.3 \times 10^{01}$ \\
\hline$Q_{\mathrm{d}}$ at $s>1 \mathrm{~mm}$ & $4.0 \times 10^{05}$ & $8.1 \times 10^{05}$ & $6.9 \times 10^{05}$ & $2.1 \times 10^{05}$ & $1.2 \times 10^{04}$ \\
\hline$Q_{\mathrm{d}}$ at $s>750 \mu \mathrm{m}$ & $8.0 \times 10^{05}$ & $1.6 \times 10^{06}$ & $1.5 \times 10^{06}$ & $5.2 \times 10^{05}$ & $3.2 \times 10^{04}$ \\
\hline$Q_{\mathrm{d}}$ at $s>500 \mu \mathrm{m}$ & $2.2 \times 10^{06}$ & $4.3 \times 10^{06}$ & $4.5 \times 10^{06}$ & $1.7 \times 10^{06}$ & $1.3 \times 10^{05}$ \\
\hline$Q_{\mathrm{d}}$ at $s>250 \mu \mathrm{m}$ & $1.1 \times 10^{07}$ & $2.3 \times 10^{07}$ & $2.9 \times 10^{07}$ & $1.3 \times 10^{07}$ & $1.5 \times 10^{06}$ \\
\hline$Q_{\mathrm{d}}$ at $s>100 \mu \mathrm{m}$ & $1.0 \times 10^{08}$ & $2.0 \times 10^{08}$ & $3.5 \times 10^{08}$ & $2.1 \times 10^{08}$ & $3.7 \times 10^{07}$ \\
\hline$Q_{\mathrm{d}}$ at $s>50 \mu \mathrm{m}$ & $5.5 \times 10^{08}$ & $1.1 \times 10^{09}$ & $2.2 \times 10^{09}$ & $1.7 \times 10^{09}$ & $4.2 \times 10^{08}$ \\
\hline$Q_{\mathrm{d}}$ at $s>30 \mu \mathrm{m}$ & $1.8 \times 10^{09}$ & $3.7 \times 10^{09}$ & $8.9 \times 10^{09}$ & $7.7 \times 10^{09}$ & $2.5 \times 10^{09}$ \\
\hline$Q_{\mathrm{d}}$ at $s>15 \mu \mathrm{m}$ & $9.5 \times 10^{09}$ & $1.9 \times 10^{10}$ & $5.8 \times 10^{10}$ & $6.2 \times 10^{10}$ & $2.8 \times 10^{10}$ \\
\hline$Q_{\mathrm{d}}$ at $s>10 \mu \mathrm{m}$ & $2.5 \times 10^{10}$ & $5.1 \times 10^{10}$ & $1.7 \times 10^{11}$ & $2.1 \times 10^{11}$ & $1.2 \times 10^{11}$ \\
\hline$Q_{\mathrm{d}}$ at $s>4 \mu \mathrm{m}$ & $2.3 \times 10^{11}$ & $4.6 \times 10^{11}$ & $2.1 \times 10^{12}$ & $3.2 \times 10^{12}$ & $2.9 \times 10^{12}$ \\
\hline$Q_{\mathrm{d}}$ at $s>2 \mu \mathrm{m}$ & $1.2 \times 10^{12}$ & $2.4 \times 10^{12}$ & $1.3 \times 10^{13}$ & $2.6 \times 10^{13}$ & $3.2 \times 10^{13}$ \\
\hline$Q_{\mathrm{d}}$ at $s>1 \mu \mathrm{m}$ & $6.5 \times 10^{12}$ & $1.3 \times 10^{13}$ & $8.7 \times 10^{13}$ & $2.1 \times 10^{14}$ & - \\
\hline$Q_{\mathrm{d}}$ at $s>0.435 \mu \mathrm{m}$ & $4.8 \times 10^{13}$ & $9.5 \times 10^{13}$ & $8.2 \times 10^{14}$ & $2.5 \times 10^{15}$ & - \\
\hline$Q_{\mathrm{d}}$ at $s>0.203 \mu \mathrm{m}$ & $2.9 \times 10^{14}$ & $5.9 \times 10^{14}$ & $6.4 \times 10^{15}$ & $2.5 \times 10^{16}$ & - \\
\hline$Q_{\mathrm{d}}$ at $s>0.0942 \mu \mathrm{m}$ & $1.9 \times 10^{15}$ & $3.7 \times 10^{15}$ & $5.1 \times 10^{16}$ & $2.5 \times 10^{17}$ & - \\
\hline$Q_{\mathrm{d}}$ at $s>0.0437 \mu \mathrm{m}$ & $1.2 \times 10^{16}$ & $2.4 \times 10^{16}$ & $4.1 \times 10^{17}$ & - & - \\
\hline$Q_{\mathrm{d}}$ at $s>0.0203 \mu \mathrm{m}$ & $7.7 \times 10^{16}$ & $1.5 \times 10^{17}$ & $3.2 \times 10^{18}$ & - & - \\
\hline
\end{tabular}

in Table 3 must still be lower limits to the observed $A f \rho_{\mathrm{o}}$ values. To compute a realistic upper limit to the maximum grain size that can be lifted from the nucleus of 67P, it is necessary to know the 3D shape of the 67P nucleus. Weiler et al. (2004b) obtain ejectable sizes up to $10 \mathrm{~cm}$, but their performed $1 \mathrm{D}$ computations are affected by large uncertainties. Therefore, we prefer to stop the integration of Eq. (6) at $s_{2}=1 \mathrm{~cm}$ at all SunComet distances. This assumption affects the extrapolated dust mass loss rates, computed by integrating $\frac{4}{3} \pi \rho_{\mathrm{d}} s^{3} \mathrm{~d} Q_{\mathrm{d}}$ over all possible dust sizes. Before perihelion only, such a computation provides loss rates lower than those shown in Fig. 7, because in Table 3 we lose the most massive grains: anyway, the loss rate decrease after perihelion is confirmed.

The $A f \rho_{\mathrm{c}}$ values in Table 3 must be compared to $A f \rho_{\mathrm{o}}$ values to check the reliability of the outputs of the tail models, which must provide $A f \rho_{\mathrm{c}}$ values consistent with dust coma photometry after we extrapolate the sizes to all possible values. In Fig. 8 we show the available $A f \rho_{\mathrm{o}}$ data covering a whole year around two perihelion passages of $67 \mathrm{P}$, provided by CCD photometry of UAI amateur observations. The consistence between $A f \rho_{\mathrm{c}}$ values in Table 3 and the $A f \rho_{\mathrm{o}}$ values in Fig. 8 shows that the dust parameters listed in Table 3 offer a schematic summary consistent with all available 67P observations. In particular, the fact that around perihelion grains much larger than $1 \mathrm{~cm}$ were probably ejected may account for the still underestimated $A f \rho_{\mathrm{c}}$ values in Table 3 . Moreover, Af $\rho_{\mathrm{c}}=0.5 \mathrm{~m}$ at $t-T=150$ days in Table 3 is consistent

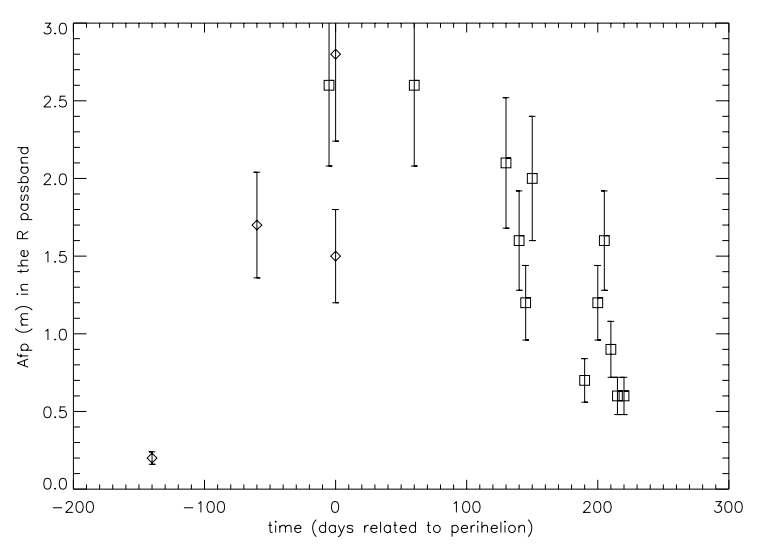

Fig. 8. $A f \rho_{\mathrm{o}}$ values obtained by means of CCD $R$-band photometry provided by the CARA network (http://cara.uai .it). Diamonds: 1996 perihelion passage. Squares: 2002 perihelion passage. The values show a clear asymmetry with respect to perihelion, with higher values after perihelion. This temporal evolution is consistent with the extrapolated $A f \rho_{\mathrm{c}}$ values computed in Table 3, supporting the outputs of the inverse tail model, and is in the opposite sense of the dust mass loss rate evolution, due to the large changes with time of the dust size distribution.

with $A f \rho_{\mathrm{o}}=0.4 \mathrm{~m}$ observed by Lamy et al. (2003) at $t-T=$ 206 days after perihelion, while $A f \rho_{\mathrm{c}}=0.1 \mathrm{~m}$ at $2 \mathrm{AU}$ before perihelion is consistent with $A f \rho_{\mathrm{o}}=0.16 \mathrm{~m}$ observed at $3 \mathrm{AU}$ 
after perihelion (Schulz 2003). Only a time-dependent index $\alpha$ can make consistent the outputs of the tail model (Fig. 7) with the $A f \rho_{\mathrm{o}}$ values (Fig. 8): a time-independent dust size distribution with $\alpha=-3.5$ at $\mathrm{mm}$ sizes and $\alpha=-4.5$ at micron sizes would be consistent with the results shown in Fig. 7, but would provide $A f \rho_{\mathrm{c}}$ values much higher than $A f \rho_{\mathrm{o}}$ at $t-T>100$ days.

\section{Future models of the 67P nucleus}

The temporal evolution of all the quantities shown in Fig. 7 suggests seasons of a comet nucleus characterized by an inhomogeneous surface, following a similar interpretation of the outputs of the same tail model applied to Comet 2P/Encke (Epifani et al. 2001). Let us assume that both the obliquity of the spin axis of the nucleus and its angle with the perihelionaphelion line are closer to $90^{\circ}$ than to zero: then, one hemisphere of the nucleus (let us call it the northern one) will be the only one to be exposed to Solar radiation before perihelion, while after perihelion only the southern one will be heated by the Sun. During the 1982 perihelion passage, the gas production rates (A'Hearn et al. 1995; Cochran et al. 1992) for $\mathrm{OH}$, $\mathrm{CN}, \mathrm{C}_{3}, \mathrm{C}_{2}$ and $\mathrm{NH}$ were systematically higher after perihelion than before. Within our hypothesis, this gas loss asymmetry suggests that the ice fraction of the northern hemisphere is larger than that of the southern one. The output shown in Fig. 7 requires that the dust covering the two hemispheres is characterized by different physical parameters. For instance, if the northern hemisphere is characterized by a dust size distribution with many more $\mathrm{mm}$ and $\mathrm{cm}$-sized grains than the southern one, we directly explain the temporal evolution of the index $\alpha$ in Fig. 7. This scenario implies that the temporal evolution of the dust mass loss rate becomes opposite to the temporal evolution of the gas loss rates and of the dust coma brightness: while the dust mass loss rate reaches the largest values before perihelion, the dust coma brightness and the gas loss rates reach the largest values after perihelion.

The temporal evolution of the dust ejection velocity in Fig. 7 seems to require a relationship between the dust size and the $\beta$ parameter which is more complex than Eq. (1). The $\beta$ parameter describes the ratio between solar radiation pressure and gravity forces on a dust grain, so that it depends on the ratio between the grain cross section and mass. However, the gas drag efficiency on a grain also depends on the ratio between its cross section and mass. The dust velocities in Fig. 7 are lower post- than pre-perihelion, despite higher gas coma densities after perihelion than before. This apparent contradiction suggests that realistic models of the drag due to gas and that due to solar radiation pressure should also take into account the spin state of non-spherical grains. $\beta$ might depend on the grain shape and spin state, which may be different in the coma and in the tail. This fact does not affect the outputs of the tail models adopted in this paper, which are all related to $\beta$ values: it simply suggests that the conversion from $\beta$ to dust sizes is more complex than Eq. (1).

The proposed seasonal scenario will remain speculative until 3D models of the thermal evolution of the 67P nucleus will be available: these are impossible until the shape and spin state of the nucleus of $67 \mathrm{P}$ have been defined. The spin state we have suggested here will be used as input of $3 \mathrm{D}$ thermal models of the $67 \mathrm{P}$ nucleus, assuming that the torques due to the gases leaving the surface change this state on timescales much longer than the comet orbital period. If this is the case, nucleus photometry performed during the aphelion might constrain the spin state proposed here. We cannot exclude other explanations, based on a homogeneous nucleus where dust fragmentation is active after perihelion only. Also realistic models able to describe this second possibility require detailed information of the nucleus shape, topography and spin state, i.e. the same information required by the seasonal scenario we are proposing here. No realistic dust fragmentation model in a realistic coma is now available (Crifo \& Rodionov 1999).

If $3 \mathrm{D}$ thermal models of the 67P nucleus confirm that the proposed seasons can explain the time evolution of the gas loss rates, we still have to explain how these seasons affect the mass dust loss rate during the whole $67 \mathrm{P}$ orbit. The proposed seasonal scenario predicts that the mass loss rate remains at the low post-perihelion values (about $10 \mathrm{~kg} \mathrm{~s}^{-1}$ ) during all the postperihelion times, possibly decreasing towards the aphelion. The Neck-Line data suggest that at 3.6 AU pre-perihelion the mass loss rate increases from $10 \mathrm{~kg} \mathrm{~s}^{-1}$ (or much less according to which dust size CO and water can lift up) to the $100 \mathrm{~kg} \mathrm{~s}^{-1}$ typical of the pre-perihelion times in Fig. 7. This is due to the size distribution of the grains covering (in our speculative scenario) the northern hemisphere, which is dominated by $\mathrm{mm}$ and $\mathrm{cm}$-sized grains. Neck-Line data require that the gas at 3.6 AU pre-perihelion is dense enough to lift these large grains. If the nucleus is a sphere with $\rho_{\mathrm{n}}=10^{3} \mathrm{~kg} \mathrm{~m}^{-3}$ and ejects at least $5 \times 10^{27} \mathrm{~s}^{-1} \mathrm{CO}$ molecules, then $\mathrm{cm}$-sized spheres with $\rho_{\mathrm{d}}=10^{3} \mathrm{~kg} \mathrm{~m}^{-3}$ are ejected all around the comet orbit, but will dominate both dust mass and coma brightness before perihelion only, due to the temporal evolution of the dust size distribution. IRAM observations (Bockelee-Morvan et al. 2004) performed at $3 \mathrm{AU}$ after 2002 perihelion gave $Q_{\mathrm{CO}}<1.6 \times 10^{27} \mathrm{~s}^{-1}$, but this result cannot be extrapolated to the corresponding distances before perihelion: other observations are required when the comet will reach 3.6 AU before perihelion. If the total gas loss rate (water and $\mathrm{CO}$ ) at 3.6 AU before perihelion will turn out to be much lower than the required $5 \times 10^{27} \mathrm{~mol} \mathrm{~s}^{-1}$, then the Neck-Line data suggest that both $\rho_{\mathrm{d}}$ and $\rho_{\mathrm{n}}$ may be much lower than $10^{3} \mathrm{~kg} \mathrm{~m}^{-3}$, and/or that the nucleus and/or the dust grains are non-spherical. Crifo et al. (2004) have recently computed a $3 \mathrm{D}$ model of a gas coma surrounding a non-spherical 67P nucleus (with $\rho_{\mathrm{d}}=\rho_{\mathrm{n}}=10^{2} \mathrm{~kg} \mathrm{~m}^{-3}$ ) ejecting $10^{27} \mathrm{CO} \mathrm{mol} \mathrm{s}$ and $2.5 \times 10^{26}$ water mol s${ }^{-1}$ at $3 \mathrm{AU}$ : this coma ejected dust spheres of $5 \mathrm{~cm}$ radius.

The IRAS trail data collected by Sykes \& Walker (1992) can be compared with the dust environment obtained in this paper. They assume that the dust sizes dominating the trail brightness are characterized by $\beta=10^{-3}$. After we correct this $\beta$ value to the more proper value $\beta=10^{-4}$ (see Fig. 5), we find that the total dust mass in the trail is $3 \times 10^{9} \mathrm{~kg}$. Sykes \& Walker (1992) conclude that the very short 67P trail must have been ejected during the previous perihelion passage only: according to the seasonal scenario we are proposing here, we can assume that all the trail mass was ejected from 3.6 to $1.3 \mathrm{AU}$ 
before perihelion, i.e. during one year only before perihelion (see Table 1). Then, the total IRAS 67P trail mass yields exactly the dust loss rate of $100 \mathrm{~kg} \mathrm{~s}^{-1}$ obtained in this paper.

\section{Conclusions}

During its approach to its target comet, the Rosetta probe will face a dust environment dominated both in mass and in brightness by $\mathrm{cm}$-sized grains released at a rate of $100 \mathrm{~kg} \mathrm{~s}^{-1}$. The previous Rosetta target, 46P/Wirtanen, was characterized by a dust mass loss rate 100 times lower at $2.5 \mathrm{AU}$ before perihelion, but very similar at perihelion (Fulle 2000): the seasonal scenario proposed in the previous section might explain this strong difference in terms of a dust-to-gas ratio in 67P much higher than in 46P. This explanation is consistent with the similar water loss rate and the different nucleus sizes of $67 \mathrm{P}$ and $46 \mathrm{P}$ (4 km vs. $1.5 \mathrm{~km}$, Lamy 2003). However, all these facts do not help us to define what is a representative comet (Weissman 1999).

The extrapolations given in Table 3 allow us to compute the number $N_{\text {lay }}$ of layers per day of dust grains larger than two microns (for smaller grains the extrapolations may become arbitrary) deposited on a lander at the nucleus surface:

$N_{\text {lay }}=\frac{s_{1}^{2}(\alpha+1) Q_{\mathrm{d}} \Delta t}{4(\alpha+3) R_{\mathrm{n}}^{2}}$

where $s_{1}=2 \mu \mathrm{m}, Q_{\mathrm{d}}$ and $\alpha$ are reported in Table 3, $\Delta t=$ $8.64 \times 10^{4} \mathrm{~s}$ and $R_{\mathrm{n}}=2 \mathrm{~km}$ is the nucleus radius (Lamy et al. 2003). The number of layers may be overestimated because we consider a sticking coefficient equal to one, while it is probable that not all grains will stick on the probe surfaces. Moreover, the lander itself will shadow some of the surface below it, thus stopping the water (not the $\mathrm{CO}$ ) gas release. On the other hand, we are neglecting the high flux of large grains falling on the lander from above (Crifo et al. 2004). We find that already at the Rosetta lander delivery planned at $3 \mathrm{AU}$, a lander may collect a monolayer of grains larger than two microns every five days, while the Rosetta probe orbiting at a distance of $10 \mathrm{~km}$ from the nucleus will collect the first monolayer of grains larger than two microns after the first four months.

Table 3 is a comprehensive summary of the dust environment of 67P consistent with all available observations. The dust environment from 3.6 $\mathrm{AU}$ to perihelion, i.e. during all the time the Rosetta probe will spend orbiting around the comet, can be summarized by the following eight points: (i) grains larger than $1 \mathrm{~cm}$ are ejected from the coma; (ii) the mass loss rate is larger than $100 \mathrm{~kg} \mathrm{~s}^{-1}$; (iii) the mass loss rate is dominated by the largest ejected grains; (iv) the dust coma brightness (e.g. the Af $\rho$ quantity) depends significantly or even mainly upon the largest ejected dust grains; (v) a low $A f \rho=0.1 \mathrm{~m}$ is consistent with such a scenario; (vi) the first dust monolayer will be collected by the lander in the first week; (vii) the first dust monolayer will be collected by the orbiter in the first four months spent at distances less than $10 \mathrm{~km}$ from the nucleus; (viii) since the dust brightness is dominated by, or at least significantly depends upon the largest ejected grains, spurious identifications of stars by the navigation cameras is a primary danger for the mission: robust software against false nucleus detection is mandatory.

\section{References}

A'Hearn, M. F., Schleicher, D. G., Feldman, P. D., Millis, R. L., \& Thompson, D. T. 1984, AJ, 89, 579

A'Hearn, M. F., Millis, R. L., Schleicher, D. G., Osip, D. J., \& Birth, P. V. 1995, Icarus, 118, 223

Bockelee-Morvan, D., Moreno, R., Biver, N., et al. 2004, Proc. of The New Rosetta Targets Meeting, in press

Brandt, J. C., Roosen, R. G., \& Harrington, R. S. 1972, ApJ, 177, 277

Burns, J. A., Lamy, P. L., \& Soter, S. 1979, Icarus, 40, 1

Cochran, A. L., Barker, E. S., Ramseyer, T. F., \& Storrs, A. D. 1992, Icarus, 98, 151

Cremonese, G., \& Fulle, M. 1989, Icarus, 80, 267

Crifo, J. F., \& Rodionov, A. V. 1999, Plan. Space Sci., 47, 797

Crifo, J. F., Lukyanov, G. A., Zakharov, V. V., \& Rodionov, A. V. 2004, Proc. of The New Rosetta Targets Meeting, in press

Epifani, E., Colangeli, L., Fulle, M., et al. 2001, Icarus, 149, 339

ESA 2003, Proc. of the 12th RSWT, 13 Feb. 2003

Fulle, M. 1988, A\&A, 201, 161

Fulle, M. 1989, A\&A, 217, 283

Fulle, M. 1999, Plan. Space Sci., 47, 827

Fulle, M. 2000, Icarus, 145, 239

Fulle, M., \& Sedmak, G. 1988, Icarus, 74, 383

Fulle, M., Bosio, S., Cremonese, G., et al. 1993, A\&A, 272, 634

Kimura, H., \& Liu, C. P. 1977, Chin. Astron., 1, 235

Lamy, P. L., Toth, I., Weaver, H., Jorda, L., \& Kaasalainen, M. 2003, A\&AS, DPS 35, 30.04

Pansecchi, L., Fulle, M., \& Sedmak, G. 1987, A\&A, 176, 358

Reach, W. T., Hicks, M. D., Gillam, S., et al. 2003, A\&AS, DPS 35, 30.07

Schulz, R. 2003, Abstract Book of The New Rosetta Targets Meeting, 10

Sekanina, Z., \& Schuster, H. E. 1978, A\&A, 65, 29

Sykes, M. V., \& Walker, R. G. 1992, Icarus, 95, 180

UAI 2003, Comet Archive, http://comete.uai.it/67p/index.htm

Weiler, M., Rauer, H., \& Helbert, J. 2004a, A\&A, 414, 749

Weiler, M., Knollenberg, J., \& Rauer, H. 2004b, Proc. of The New Rosetta Targets Meeting, in press

Weissman, P. 1999, in Composition and Origin of Cometary Materials, ed. K. Altwegg et al. (Kluwer), 301

Yeomans, D. K., \& Wimberly, R. N. 1991, in Comets in the PostHalley Era, ed. R. L. Jr. Newburn et al., 2, 1281 